\title{
Increased intracranial pressure in myelomeningocele (MMC) patients never shunted: Results of a prospective preliminary study
}

\author{
J Iborra*, $^{*}$, E Pagès ${ }^{1}$, A Cuxart ${ }^{1}$, A Poca ${ }^{2}$ and J Sahuquillo ${ }^{2}$ \\ ${ }^{1}$ Spina Bifida Unit, Vall d'Hebron Hospitals, Barcelona, Spain; ${ }^{2}$ Department of Neurosurgery, Vall d'Hebron \\ Hospitals, Barcelona, Spain
}

Study design: In some young adults non-shunted myelomeningocele (MMC) patients without symptoms or with unspecific clinical findings, moderate or severe stabilised ventriculomegaly are observed in control CT scans. Physicians are then faced with the so-called 'Arrested Hydrocephalus' (AH) syndrome. The present study is part of a prospective protocol in which patients with hydrocephalus associated with MMC and long-term clinical diagnosis of $\mathrm{AH}$ were included.

Objectives: To evaluate myelomeningocele patients never shunted with clinical and/or CT scan criteria compatible with AH.

Setting: Multidisciplinary Spina Bifida Unit in a tertiary university hospital in Barcelona, Spain.

Methods: Fourteen MMC patients were selected, in all of them, continuous intracranial pressure (ICP) monitoring was performed. Analysis of the ICP records was done using the method described by Børgesen in $\mathrm{mmHg}$. All patients were also studied by intelligence quotient (IQ) testing before and after shunting.

Results: The CT in all the study cases showed an Evans ratio $\leqslant 0.30$. The majority of patients (11/14) presented continuous high or intermittently raised ICP (active or unstable compensated hydrocephalus group) and in three (3/14) cases we confirmed the diagnosis of arrested hydrocephalus. There were no complications associated with ICP monitoring.

Conclusions: In view of our results, we believe that in all non-shunted cases with clinical and/ or CT scan criteria compatible with $\mathrm{AH}$, the use of an objective method, such as continuous ICP monitoring, is necessary to diagnose arrested hydrocephalus, and a more comprehensive method that includes extensive neuropsychological valoration should be developed for the diagnosis and treatment of arrested hydrocephalus.

Sponsorship: Supported in part by Grant number 97-0923 from the Fondo de Investigaciones Sanitarias de la Seguridad Social (FISS).

Spinal Cord (2000) 38, 495-497

Keywords: myelomeningocele; intracranial pressure; arrested hydrocephalus; cerebrospinal fluid shunts

\section{Introduction}

Myelomeningocele (MMC) is a complex syndrome that affects mainly the nervous, musculoskeletal and genitourinary systems. Over the last few decades survival and quality of life has increased in MMC patients.

Most children born with MMC will have associated hydrocephalus but the current percentage of children partially depends on the working definition. If hydrocephalus is defined as any degree of ventriculomegaly, less than $5 \%$ of these infants are spared hydrocephalus. ${ }^{1}$ While some children born with MMC have overt hydrocephalus most are born with normal

*Correspondence: J Iborra, Spina Bifida Unit, Hospital Universitari de Traumatologia i Rehabilitació, Vall d'Hebron Hospitals, P/Vall d'Hebron 119-129, 08035 Barcelona, Spain or slightly smaller than normal heads and normal or slightly generous ventricles. In most infants, the need for shunting is quickly apparent; however, in 20 to $30 \%$, the degree of ventriculomegaly is only mild compared to controls and the rate of head growth tends to increase initially and to follow normal patterns afterwards. ${ }^{1-3}$

These patients reach mature life without a shunt, and in some of them without symptoms or with minimum non-specific signs. Moderate or severe stabilised ventriculomegaly is observed in control CT scans. Physicians are then faced with so-called 'Arrested Hydrocephalus' (AH). ${ }^{4-6}$ Some authors have given warnings about the severe problems that patients with $\mathrm{AH}$ can present. AH has not been systematically studied in MMC patients. An increase 
in intracranial hypertension can result in irreversible injury in $\mathrm{CNS}^{7}$ The potential for late deterioration in patients with $\mathrm{MMC}$ and $\mathrm{AH}$ creates a burden of responsibility on their caretakers.

We report the results of a prospective study of 14 never shunted MMC patients, with clinical and/or CT scan criteria compatible with $\mathrm{AH}$. In all of them, continuous ICP monitoring was performed.

\section{Patients and methods}

The present study is part of a prospective protocol in which patients with hydrocephalus associated to MMC and long-term clinical diagnosis of $\mathrm{AH}$ were included. For admission to this protocol the following criteria were necessary: (1) MMC patients treated and followed in our Unit; (2) they had never been shunted or received any treatment for hydrocephalus; (3) no evidence of progressive increase in ventricular size in sequential CT scans over a long period of time and (4) no evident signs or symptoms of intracranial hypertension.

All patients underwent continuous intracranial pressure (ICP) monitoring using a fiberoptic extradural device (LADD Research Industries, Inc., Burlington, Vermont, USA) introduced through a pre-coronal burr hole. In all cases ICP monitoring was performed for at least $72 \mathrm{~h}$ including overnight recording. Hard copies of the ICP were obtained through a single channel recorder with a paper speed of $6-20 \mathrm{~cm} / \mathrm{h}$. The patient was maintained lying down throughout the recording time. The analysis of the ICP records were made using the method described by Børgesen ${ }^{8}$ in $\mathrm{mmHg}$.

Mean resting ICP was calculated from the records every $15 \mathrm{~min}$ in periods without fluctuations. Amplitude of pulse waves in stable periods of recording and the presence of high amplitude B-waves $(1 / 2-12 / \mathrm{min}$ ICP fluctuations more than $10 \mathrm{mmHg}$ above baseline and lasting at least $10 \mathrm{~min}$ ), were evaluated. Low amplitude B-waves were considered when they had an amplitude less than $10 \mathrm{mmHg}$ above base line. Awaves were considered to be present when constant pressure elevations at least $20 \mathrm{mmHg}$ above the resting line with abrupt onset and end, and lasting at least $10 \mathrm{~min}$ were observed. To avoid miscalculation due to the different durations of the monitoring time, plateau and B-waves were expressed as a percentage of the first $24 \mathrm{~h}$ period of the total recording time. ${ }^{9}$

According to mean ICP and the percentage of high amplitude B-waves, patients were classified in the following categories. (1) Active hydrocephalus (mean ICP $>12 \mathrm{mmHg}$ ). (2) Compensated unstable hydrocephalus (mean ICP $\leqslant 12 \mathrm{mmHg}$ and $\mathrm{B}$-waves present in more than $25 \%$ of the recording time). (3) Compensated stable hydrocephalus (mean ICP $\leqslant 12 \mathrm{mmHg}$ and B-waves present in less than $25 \%$ of the total recording time). (4) Arrested hydrocephalus (mean $\mathrm{ICP} \leqslant 12 \mathrm{mmHg}$ without any pathological wave activity). ${ }^{9}$
In all patients a $\mathrm{CT}$ scan was performed and the measurement of Evans index $(E I=$ Maximum bifrontal distance/Maximum inner diameter of the skull at the level of the measurement of the maximum bifrontal distance) was calculated. The WISC-R was administered to each patient under 17-years-of-age and the WISA-R was given to patients aged 17 years and older (before shunt and at 1 year after shunt). Three Wechsler scores were obtained for each patient: a verbal IQ, a performance IQ, and a full-scale IQ. The full-scale IQ was determined by averaging scores obtained from the verbal and performance IQs.

\section{Results}

Nine males and five females, aged 13-35 years (average $\pm \mathrm{SD}, 21.9 \pm 6$ years) were studied. Two of these patients had a thoracic level, one a mid lumbar, four a low lumbar and seven had a sacral level. From a clinical point of view, five patients were completely asymptomatic and the other nine cases showed a history of clinical findings that were not considered directly related to the hydrocephalus (4/9 headache that occasionally needed the use of minor analgesics and did not interfere with activities of daily living, 1/9 behaviour changes (impulsivity, inappropriate sexual comments and behaviour, behavioural inertia), 2/9 lower extremities spasticity with tethered cord associated, and 2/9 impaired competence in school performance and social function).

In the cognitive status patients ranged in IQ from $61-107$ with a mean IQ of $85.2 \pm 12.9$. The mean head circumference of the study group was $60 \pm 1.5 \mathrm{~cm}$ (range $57-63 \mathrm{~cm}$ ) and the CT scan in all cases showed an Evans ratio $\geqslant 0.30$.

Continuous recording of the ICP showed in four patients an elevated mean ICP and they were included in the active hydrocephalus group. Seven cases presented an important B activity and were included in the compensated hydrocephalus group (two unstable and five stable). In three patients the continuous ICP monitoring showed a normal value during all the recording and no pathological waves, which confirmed the diagnosis of arrested hydrocephalus (Table 1).

All patients with an active or compensated hydrocephalus were shunted except one patient with stable compensated hydrocephalus that didn't want it. No patient with arrested hydrocephalus was shunted. At 1 year post shunting no significant differences in IQ scores were found. There were no complications associated with ICP monitoring and there was only one patient with shunt overdrainage (subdural haematoma).

\section{Conclusions}

Patients with hydrocephalus associated with MMC are difficult to manage. Most patients born with MMC will need shunting. Significant controversy exists about how 
Table 1 Summary of ICP and the different types of hydrocephalus

\begin{tabular}{|c|c|c|c|c|c|c|}
\hline & $n=14$ & $\begin{array}{c}\text { Mean age } \\
\text { (Range) years }\end{array}$ & $\begin{array}{c}\text { Mean } \\
\text { ICP } m m H g\end{array}$ & A-waves & $\begin{array}{c}\text { High } \\
\text { B-waves }\end{array}$ & $\begin{array}{c}\text { Low } \\
\text { B-waves }\end{array}$ \\
\hline Active & 4 & $19(13-24)$ & 16.2 & $2 / 4$ & $4 / 4$ & $4 / 4$ \\
\hline C. Unstable & 2 & $23(18-28)$ & 6.5 & $0 / 2$ & $2 / 2$ & $2 / 2$ \\
\hline C. Stable & 5 & $25.8(19-35)$ & 10.6 & $0 / 5$ & $5 / 5$ & $5 / 5$ \\
\hline Arrested & 3 & $18.3(15-24)$ & 9 & $0 / 3$ & $0 / 3$ & $0 / 3$ \\
\hline
\end{tabular}

to select patients with stabilised ventriculomegaly who can benefit from a shunt. When faced with adult patients without shunt and non-neuroradiological evidence of hydrocephalus progression it is difficult to differentiate between a compensated and arrested hydrocephalus. On the other hand, the concept of arrested hydrocephalus has been always controversial. $^{4-6,10}$ In the literature, the prevalence of spontaneously arrested hydrocephalus has been described as variable as $9 \%$ to $47 \%$ of patients. ${ }^{11-13}$

Studies of intellectual functioning of children with MMC have shown that children who never had been shunted, on the whole, do better than shunted children. ${ }^{14,15}$ Interpreting such retrospective studies to help make decisions on individual patients leads to difficulty because the shunted group presumably had more severe hydrocephalus initially. The biologic cost of untreated hydrocephalus must be evaluated. Thus, when ICP increases equal to or above $20 \mathrm{mmHg}$, neuronal injury may develop. The major pathophysiologic problems associated with increased ICP are ischemia and herniation. ${ }^{6}$ Single IQ scores are inadequate measurements of intellectual function in young adult $\mathrm{MMC}$ patients with $\mathrm{AH}^{10}$ and serial examinations should be carried out. Recent studies have tended to show that more subtle neuropsychological parameters not measured on IQ tests may be improved with normal or smaller than normal ventricles. $^{16-19}$ Neuropsychological assessment included test of verbal and visual memory, visuospatial functions, speed of mental processing and frontal lobe functions. In these cases apart from major problems hydrocephalus related, cognitive or behavioural problems can handicap and compromise quality of life.

In view of our preliminary results, we believe that in all cases with clinical and/or CT scan criteria compatible with $\mathrm{AH}$, the use of an objective method, such as continuous ICP monitoring, is necessary to diagnose arrested hydrocephalus, and a more comprehensive method that included extensive neuropsychological valoration should be developed for the diagnosis and treatment of arrested hydrocephalus.

\section{References}

1 Rekate HL. Management of hydrocephalus in the newborn. In: Rekate HL (ed). Comprehensive management of spina bifida. CRC Press Inc. Boca Raton. Florida 1991, pp 16-26.
2 Iborra J. Deformidades del raquis en el mielomeningocele. Estudio de prevalencia y de historia natural. Tesis Doctoral. Universitat Autónoma de Barcelona. Facultat de Medicina, Bellaterra, Spain, 1995.

3 Stein SC, Schut L. Hydrocephalus in myelomeningocele. Childs Brain 1979; 5: $413-419$.

4 Schick RW, Matson DD. What is arrested hydrocephalus?. $J$ Pediatr 1961; 58: $791-799$.

5 Bird MD, Ratcheson RA, Seigel BA, Fishman MA. The evaluation of arrested communicating hydrocephalus utilizing cerebrospinal fluid dynamics: a preliminary report. Dev Med Child Neurol 1973; 15: 474-482.

6 McLone DG, Aronyk KE. An approach to the management of arrested and compensated hydrocephalus. Pediatr Neurosurg 1993; 19: $101-103$.

7 Lyons MK, Meyer FB. Cerebrospinal fluid physiology and the management of increased intracranial pressure. Mayo Clin Proc 1990; 65: $684-707$.

8 Børgesen SE, Gjerris F, Sorensen SC. Intracranial pressure and conductance to outflow of cerebrospinal fluid in normal-pressure hydrocephalus. J Neurosurg 1979; 50: $489-493$.

9 Sahuquillo J, Rubio E, Codina A, Molins A, Guitart JM, Poca MA, Chasampy A. Reappraisal of the intracranial pressure and cerebrospinal fluid dynamica in patients with the so-called 'Normal pressure hydrocephalus' syndrome. Acta Neurochir (Wien) 1991; 112: 50-61.

10 Hammock MK, Milhorat TH, Baron IS. Normal pressure hydrocephalus in patients with myelomeningocele. Dev Med Child Neurol 1976; 37(Suppl): 55-68.

11 DiRocco C, Caldarelli M, Giulio M, Rossi GF. The study of cerebrospinal fluid dynamica in apparently 'arrested' hydrocephalus in children. Childs Brain 1977; 3: $359-374$.

12 Hemmer R, Böhm B. Once a shunt, always a shunt? Dev Med Child Neurol (suppl) 1976; 18: 69-73.

13 Holzer GJ, de Lange SA. Shunt-independent arrest of hydrocephalus. J Neurosurg 1973; 39: 689-701.

14 Mapstone TB et al. Relationship of CSF shunting and IQ in children with myelomeningocele: a retrospective analysis. Childs Brain 1984; 11: 112 - 118 .

15 Soare PL, Raimondi AJ. Intellectual and perceptual-motor characteristics of treated myelomeningocele children. Am J Dis Child 1977; 131: 199-204.

16 Torkelson RD, Leibrock LG, Gustavson JL, Sundell RR. Neurological and neuropsychological effects of cerebral spinal fluid in children with assumed arrested ('normal pressure') hydrocephalus. J Neurosurg Psyquiatry 1985; 48: $799-806$.

17 Thompson NM et al. Cognitive and motor abilities in preschool hydrocephalics. J Clin Exp Neuropsychol 1991; 13: 245-258.

18 Bier JC et al. Medical and social factors associated with cognitive outcome in individuals with myelomeningocele. Dev Med Child Neurol 1997; 39: 263 - 266.

19 Morrow JD, Wachs TD. Infants with myelomeningocele: visual recognition memory and sensorimotor abilities. Dev Med Child Neurol 1992; 34: 488 - 498. 